\title{
USO DE ANSIOLITICOS: ABUSO OU NECESSIDADE?
}

\author{
USE OF ANSIOLITICS: ABUSE OR NEED?
}

\section{Viviane Rosset FÁVERO'1, Marcelo del Olmo SATO², Ronise Martins SANTIAGO³}

1 - Acadêmica do curso de Farmácia do Centro Universitário Campos de Andrade, UNIANDRADE, Curitiba, Brasil.

2 - Médico e Professor da Faculdade Evangélica do Paraná, FEFAR, Curitiba, Brasil

3 - Farmacêutica e Professora do curso de Farmácia do Centro Universitário Campos de Andrade, UNIANDRADE, Curitiba, Brasil.

Autor para correspondência:

\section{RESUMO:}

Os ansiolíticos apresentam componentes que atuam no controle da ansiedade de pacientes com alterações de saúde associadas ao Sistema Nervoso Central (SNC), afetando suas emoções e seu comportamento. Por vezes seu uso ocorre de forma abusiva, podendo gerar diversos efeitos adversos. Desta forma, objetivou-se analisar as indicações clínicas dos ansiolíticos em uma farmácia de dispensação, identificando o tempo de uso, a especialidade médica do prescritor e os medicamentos ansiolíticos mais vendidos. Para tanto, foi realizada pesquisa descritiva de abordagem quantitativa. Ao todo, participaram desta pesquisa 32 usuários, sendo a maioria do sexo feminino, com idade superior a 40 anos. O fármaco, na maioria dos casos foi indicado pelo médico clínico geral, psiquiatra ou neurologista. No entanto, o acesso aos ansiolíticos ocorreu também através de familiares ou amigos, sem prescrição médica. Quanto ao motivo do uso da medicação, destacaram-se a ansiedade, a depressão e a insônia. Em se tratando da frequência do seu uso, a maioria relatou consumi-las diariamente; porém alguns só o fazem esporadicamente. Apenas três pacientes relataram ter apresentado reações adversas. Constatou-se que muitos pacientes ao tentar interromper o seu uso, apresentaram sintomas de abstinência. Concluiu-se que o uso de ansiolíticos na maioria das vezes ocorre de forma irracional.

Palavras-chave: ansiolíticos; psicotrópicos; uso racional.

\section{ABSTRACT:}

Anxiolytics have components that act to control the anxiety of patients with health problems associated with the central nervous system, affecting their emotions and their behavior. Sometimes its use occurs in an abusive way, being able to generate diverse adverse effects. The aim of this study was to analyze the clinical indications of anxiolytics in a dispensing pharmacy, identifying the time of use, the prescriber's medical specialty and the best selling drugs. For that, a descriptive research of quantitative approach was carried out. In total, 32 users participated in this study, the majority being female, over 40 years of age. The drug, in most cases was indicated by the general practitioner, psychiatrist or neurologist. However, access to anxiolytics also occurred through family members or friends, without a prescription. Concerning the reason for the use of the medication, anxiety, depression and insomnia were noted. When it comes to the frequency of their use, most reported consuming them daily; But some only do so sporadically. Only three patients reported having had adverse reactions. It was found that many patients, when trying to stop their use, had withdrawal symptoms. It was concluded that it is of great importance the careful evaluation during the consultation to determine the most appropriate pharmacological treatment, in order to stimulate the rational use of these drugs. It is suggested the development of clinical protocols and continuing education actions of professionals involved in the prescription and supply of these drugs.

Keywords: Anxiolytics; Psychotropic medications; Rational use. 


\section{INTRODUÇÃO}

Os medicamentos psicotrópicos são substâncias que atuam no Sistema Nervoso Central (SNC), podendo desencadear alterações e dependência (CARVALHO et al., 2016). Estes medicamentos classificam-se em quatro categorias: ansiolíticos-sedativos; antidepressivos; estabilizadores do humor e antipsicóticos ou neurolépticos (FIGUEIREDO, 2015). Os ansiolíticos são medicamentos cujos componentes químicos atuam no controle da ansiedade com efeitos que incidem sobre as emoções, o humor e o comportamento (FIGUEREDO, 2012). O principal representante desta classe são os benzodiazepínicos, um dos medicamentos mais prescritos mundialmente, como o Diazepam, o Clonazepam, o Alprazolam e o Midazolan (CARVALHO et al., 2016).

A utilização de ansiolíticos pela população muitas vezes ocorre de maneira abusiva (NOTO et al., 2002). Este fato pode ocorrer devido a fatores como: erros em prescrições médicas, automedicação, dependência química e aumento das enfermidades relacionadas à psiquiatria (GRASSI e CASTRO 2012). Entretanto, os efeitos dessas substâncias, decorrentes do seu uso crônico, por meses ou anos, podem resultar na dependência química do usuário (GRUBER e MAZON, 2014), sendo que a abstinência prejudica severamente a sua vida social, devido à irritabilidade, à insônia excessiva, à sudoração, à dor no corpo a até mesmo às convulsões (CARLINI et al., 2001).

Diante dessas premissas, é possível afirmar que o uso irracional de psicotrópicos pode trazer problemas associados à intoxicação, além de outros impactos sociais e econômicos como: dificuldades no desenvolvimento do aprendizado dos pacientes; aumento de investimentos em saúde pública destinado ao tratamento de pessoas com dependência química; prejuízo das relações familiares; e o incentivo ao consumo ilícito dessas substâncias (LOPES e GRIGOLETO, 2011).

Deste modo, este artigo teve como objetivo analisar as indicações clínicas de ansiolíticos em uma farmácia de dispensação, correlacionando com o tempo de uso e a especialidade médica do prescritor.

\section{MATERIAL E MÉTODOS}

Este estudo foi realizado em duas etapas: em um primeiro instante, foram selecionadas prescrições médicas que continham medicamento da classe dos ansiolíticos no momento da dispensação. O paciente, acima de 18 anos, portador da receita de 
ansiolíticos foi convidado a participar voluntariamente do estudo, através do aceite do termo de consentimento livre e esclarecido. O mesmo ao aceitar participar respondeu ao questionário, contendo 15 perguntas relacionadas aos seus dados gerais como: idade, sexo e ao uso do medicamento.

Os resultados obtidos foram armazenados e processados através do programa Microsoft Office Excel, para posteriormente gerar os gráficos e tabelas.

A pesquisa foi aprovada pelo Comitê de Ética em Pesquisa com Seres Humanos do Centro Universitário Campos de Andrade (CEP-UNIANDRADE), sob o parecer número: 1.937.829.

\section{RESULTADOS}

Ao todo, participaram desta pesquisa 32 usuários, sendo que destes, 43,75\% $(n=14)$ eram do sexo masculino e $56,35 \%(n=18)$ do sexo feminino. A idade dos pacientes foi variável entre 18 a 70 anos, com idade média de aproximadamente 45,9 anos. Entre as mulheres, a média foi de 45,4 anos e entre os homens de 46,6 anos. Ainda em relação à faixa etária, 6,2\% dos usuários pertenciam à faixa entre 10 a 20 anos; 6,2\%, entre 21 a 30 anos; 15,6\%, entre 31 a 40 anos; 31,3\%, entre 41 a 50 anos; 21,9\% entre 51 a 60 anos; e $18,8 \%$ entre 61 a 70 anos.

Em relação à indicação do medicamento pela primeira vez, constatou-se que na maioria dos casos este foi indicado pelo médico $(84,4 \%)$.

Em se tratando da especialidade do médico que realizou a prescrição do medicamento, $47 \%$ dos casos foram prescritos por um clínico-geral; em $25 \%$, por um psiquiatra; 15,6\%, por um neurologista; o ginecologista, cardiologista, geriatra e gastroenterologista foram responsáveis por 3,1\% das prescrições, cada especialidade.

Quanto ao motivo do uso da medicação, sobressaiu-se a ansiedade (43,8\%), a depressão (53,1\%) e a insônia (62,5\%). Ressalta-se que nesta questão, os indivíduos poderiam marcar mais de uma opção.

Os dados referentes ao tempo em que os indivíduos utilizam a medicação demonstraram que, os entrevistados faziam uso dos ansiolíticos há anos, sendo 68,7\%, outros $31,3 \%$ faziam uso a meses.

Em se tratando da frequência do uso das medicações, 64,3\% dos homens relataram consumi-las diariamente; enquanto 35,7\% só o fazem quando necessário. As mulheres relataram o consumo diário em 66,7\%; e esporádico em 33,3\% dos casos. 
Quando questionados sobre as reações adversas aos medicamentos, nenhum homem relatou apresenta-las. Ao passo que 3 mulheres apresentaram sonolência e 1 apresentou perda de memória.

Os dados relativos à tentativa de parar de tomar a medicação e não conseguir revelou que $30 \%$ dos pacientes ja tentou retirar a medicação pelo menos uma vez. Entre estes indivíduos que tentaram parar a medicação, as reações mais frequentes foram 0 nervosismo, a insônia, a agitação e a inquietude, conforme evidenciado na Figura 1.

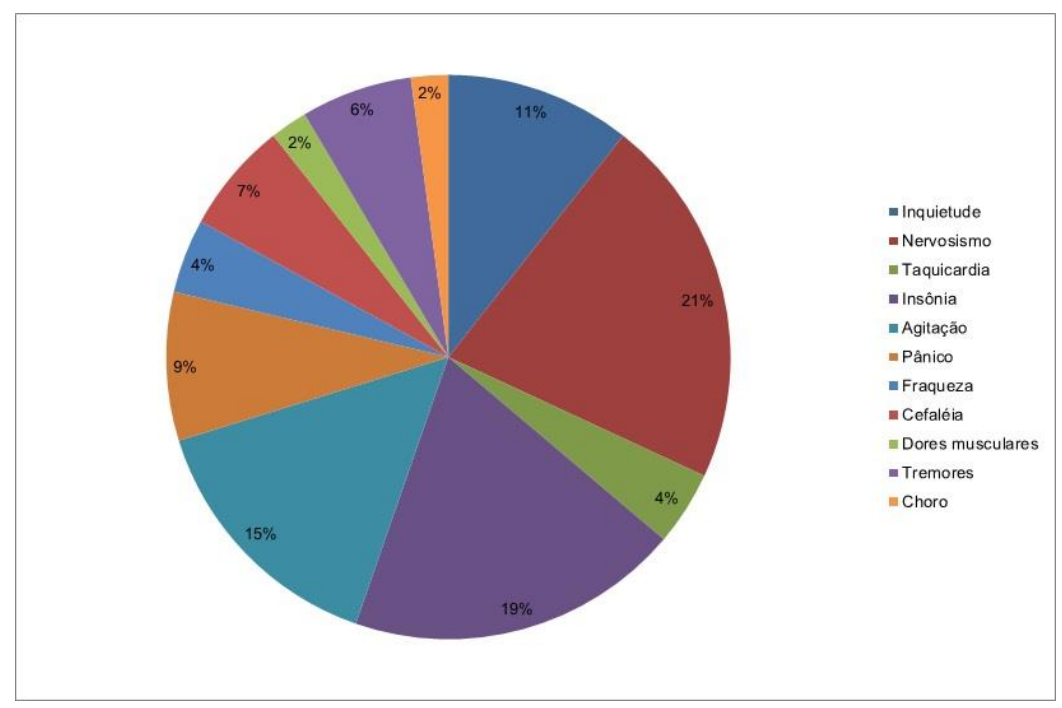

Figura 1: Reações ao tentar parar de tomar a medicação.

Quando questionados sobre já ter recebido medicamento ansiolítico de algum vizinho, parente ou amigo, 28,6\% dos homens e 22,2\% das mulheres afirmaram já ter recebido. Considerando o total de entrevistados, 25\% já haviam recebido. Em se tratando do uso de medicamento ansiolítico sem prescrição médica, 14,3\% dos homens e 16,7\% das mulheres já consumiram. Em relação ao total de entrevistados, 15,6\% confirmaram ter consumido sem prescrição.

\section{DISCUSSÃO}

Os transtornos psiquiátricos, em especial aqueles relacionados à ansiedade e depressão, têm se tornado bastante frequentes nas últimas décadas. Este aumento pode ser atribuído à maior frequência de diagnósticos na população, aos novos fármacos disponíveis no mercado farmacêutico e às novas indicações dos medicamentos existentes (ROCHA e WERLANG, 2013). 
$\mathrm{Na}$ presente pesquisa observou-se que a maioria dos pacientes era do sexo feminino, predominando aqueles com idade superior a 40 anos. Tais dados estão em consonância com outras pesquisas, Firmino e colaboradores (2011), ao avaliarem o uso de benzodiazepínicos, observaram que aproximadamente $75 \%$ das prescrições eram destinadas a mulheres e adultos. Outro estudo realizado por Yoneyama, Maruiti e Esteves (2016) analisando o uso de medicações psicotrópicas, encontrou resultados similares, com frequência de cerca de $78 \%$ em mulheres e $75 \%$ acima de 45 anos de idade.

A maior prevalência do uso desta classe medicamentosa por mulheres pode ser justificada pelo fato de serem mais preocupadas com a sua saúde, procurando mais pelos serviços de saúde; devido a maior frequência de depressão e ansiedade no gênero feminino; e também em função de fatores socioculturais (FIRMINO et al., 2011; ROCHA e WERLANG, 2013).

Em se tratando da maior frequência do uso destes medicamentos com o aumento da idade, tal fato pode ser associado a transtorno do sono, depressão e doenças neurológicas degenerativas. Além disso, em adultos o estresse no ambiente de trabalhou ou em sua vida pessoal gera maior número de transtornos e, consequentemente, maior uso de medicação (FIRMINO et al., 2011).

Os medicamentos psicotrópicos, até o fim da década de 1980, eram disponibilizados diretamente pelo farmacêutico ou atendente de farmácia, de modo que seu acesso era facilitado e a dependência era bastante frequente. Uma vez constatados os efeitos nocivos do uso indiscriminado destes fármacos, o Ministério da Saúde regulamentou o controle destas substâncias, por meio da apresentação e retenção de receita, bem como pela notificação no Sistema Nacional de Gerenciamento de Produtos Controlados (SNGPC) (AZEVEDO, 2014).

Em relação ao acesso a estes medicamentos, observou-se neste estudo que aproximadamente $16 \%$ dos participantes da pesquisa relataram ter recebido indicação do medicamento por um amigo. Além disso, muitas vezes o acesso a estes fármacos não se dá na farmácia, mas por intermédio de familiares ou amigos, como observado neste estudo, em que $29 \%$ dos homens e $22 \%$ das mulheres relataram ter obtido o medicamento desta forma. Outro dado alarmante é referente ao índice de pacientes que obtiveram o fármaco sem prescrição médica, atingindo um percentual de aproximadamente $16 \%$.

Os ansiolíticos na presente pesquisa foram prescritos majoritariamente por clínicos geral, psiquiatras ou neurologistas. Em concordância, uma pesquisa realizada por Torres e colaboradores (2014), com o propósito de analisar o uso de substâncias psicotrópicas e a 
especialidade médica do prescritor, constatou que a maioria $(31,4 \%)$ era clínico geral, $10,8 \%$ neurologistas e $7,1 \%$ psiquiatras. Tal fato pode ser atribuído ao amplo número de atendimentos realizados no âmbito da Atenção Primária à Saúde pelos clínicos geral; e pelo fato de as especialidades da neurologia e psiquiatria estarem diretamente associadas à saúde mental.

Em se tratando dos fatores que motivaram o uso da medicação a ansiedade, a depressão e a insônia foram as mais citadas. De acordo com Sweetman (2005 apud FIRMINO, 2008) os benzodiazepínicos são indicados no tratamento da ansiedade, da insônia, da epilepsia, de espasmos musculares, da síndrome de abstinência alcoólica e como adjuvante no tratamento da esquizofrenia. Nunes e Bastos (2016) destacam dentre as classes de medicamentos ansiolíticos, os benzodiazepínicos, sendo a primeira escolha para o tratamento da ansiedade e da insônia. Estes fármacos atuam como depressores do SNC, com ação ansiolítica, sedativa, miorrelaxante e anticonvulsivante.

Embora neste estudo uma das principais indicações para o tratamento com ansiolíticos, seja a depressão, Firmino (2008), reforça que os benzodiazepínicos neste caso só devem ser empregados se a ansiedade for o principal fator envolvido e na ausência de comportamento agressivo predominante. Estes medicamentos não devem ser empregados individualmente no tratamento da depressão ou ansiedade associada à depressão, uma vez que podem levar a suicídio. A autora, ao analisar receitas de benzodiazepínicos prescritos e os motivos de prescrição pelos médicos, observou que em aproximadamente $30 \%$ dos casos foi prescrito como hipnótico; em 17\% como de uso crônico/dependência; em $16 \%$ para tratamento de ansiedade; em $8 \%$ para tratamento da depressão e em $1 \%$ como anticonvulsivante (FIRMINO, 2008).

$\mathrm{Na}$ presente pesquisa constatou-se que a maioria dos pacientes faz uso da medicação ansiolítica há anos. Sabe-se que estes medicamentos devem ser empregados por um curto período de tempo, uma vez que causam dependência, tolerância e crise de abstinência quando utilizados por 4 a 6 semanas. Nos casos em que é necessário um tratamento mais longo, como o da ansiedade e da fobia, outros medicamentos com propriedades ansiolíticas e antidepressivas são indicados, como a sertralina e a paroxetina (NUNES e BASTOS, 2016).

Firmino (2008), ao analisar receitas de benzodiazepínicos prescritos, constatou que mais de $50 \%$ da amostra fazia tratamento contínuo com estes medicamentos há mais de um ano. A referida autora constatou que diversos estudos encontraram resultados semelhantes, com tratamento superior a um ano. Dados do Ministério da Saúde (1994 apud 
FIRMINO, 2008) revelam que o risco de dependência aumenta de acordo com o tempo de uso. Até um ano, o risco está entre 5 e 10\%; de 2 a 4 anos, entre 25 a $45 \%$; e acima de 4 anos, de 75\%. Apesar de haver casos em que seu uso deve ser prolongado, com cautela e acompanhamento, nota-se que frequentemente o consumo do medicamento por longos períodos de tempo não apresenta justificativa clínica plausível pelos prescritores (FIRMINO, 2008).

Quanto maior o tempo de uso da medicação, mais difícil é parar de utiliza-lo e, consequentemente, maiores são as chances de desenvolvimento de síndrome de abstinência (NUNES e BASTOS, 2016). Tal fato foi observado nesta pesquisa, uma vez que $30 \%$ dos usuários já tentaram interromper o seu uso e não conseguiram, devido ao aparecimento de sintomas, como: nervosismo, insônia, agitação, inquietude, pânico, cefaleia e tremores.

A interrupção do uso destes medicamentos não deve ser feita de forma repentina, mas gradual, sendo sua retirada feita em um período de 6 a 8 semanas (NUNES e BASTOS, 2016). Geralmente os sintomas de abstinência têm início de 5 a 10 dias após a sua retirada, incluindo: tremores, sudorese, palpitação, letargia, náusea, insônia, irritabilidade, inquietação, agitação, convulsão e alucinação (GONÇALVES, 2012).

Além das reações nos casos de abstinência, outras podem ser observadas. Quando do uso destes medicamentos em doses terapêuticas normais, reações como sonolência, confusão mental, amnésia e falta de coordenação motora podem aparecer. Em casos de superdose, podem gerar sono prolongado, porém sem depressão grave da respiração. Quando em uso prolongado, causam tolerância e dependência (CONSTANTE, 2008).

Diante dos resultados obtidos, ressalta-se a importância de que a indicação dos ansiolíticos seja realizada de forma criteriosa e fundamentada cientificamente. Além disso, é necessário que os pacientes sejam orientados acerca do uso racional desta medicação, evitando seu consumo excessivo ou sem orientação médica.

\section{CONCLUSÃO}

Observou-se que a maioria dos ansiolíticos foram prescritos por clínicos geral, psiquiatras ou neurologistas. No entanto, o acesso aos ansiolíticos diversas vezes ocorreu sem a prescrição médica e por tempo prolongado. Deste modo, concluímos que na maioria dos casos o uso dos ansiolíticos estava sendo realizada de maneira irracional. 


\section{REFERÊNCIAS}

AZEVEDO, A.J.P. Consumo privado de ansiolíticos benzodiazepínicos e sua correlação com indicadores sociodemográficos nas capitais brasileiras. $72 f$. Dissertação (Mestrado em Saúde Coletiva) - Universidade Federal do Rio Grande do Norte, Natal, 2014.

CARLINI, E. A., et al. Drogas psicotrópicas: o que são e como agem. Revista Imesc, v. 3, p. 9-35, 2001.

CARVALHO, E.F. de, et al. Perfil de dispensação e estratégias para uso racional de psicotrópicos. 45f. Monografia (Linhas de Cuidado em Enfermagem) - Universidade Federal de Santa Catarina, Florianópolis, 2016.

CONSTANTE, J. O. O perfil de uso de benzodiazepínico por usuários de uma unidade de estratégia de saúde da família de uma cidade do sul de Santa Catarina. Disponível em: < http://www.bib.unesc.net/biblioteca/sumario/00003E/00003E2B.pdf>. Acesso em: 10 mai. 2017.

FIGUEIREDO, A.C.D. de. Consumo e gastos com psicotrópicos no Sistema Único de Saúde no estado de Minas Gerais: análise de 2011 a 2013. 63f. Dissertação (Mestrado em Saúde Coletiva) - Universidade de Brasília, Brasília, 2015.

FIGUEREDO, K.C. Uso de medicamentos ansiolíticos: uma abordagem sobre o uso indiscriminado. Artigo Científico. Santa Maria: UINFRA. 2012.

FIRMINO, K.F. et al. Fatores associados ao uso de benzodiazepínicos no Serviço Municipal de Saúde da cidade de Coronel Fabriciano, Minas Gerais, Brasil. Cadernos de Saúde Pública, v.27, n.6, p. 1223-1232, 2011.

FIRMINO, K.F. Benzodiazepínicos : um estudo da indicação / prescrição no Município de Coronel Fabriciano - MG. 108 f. Dissertação (Mestrado em Ciências Farmacêuticas) Universidade Federal de Minas Gerais, Belo Horizonte, 2008.

GONÇALVES, A. L. Abuso de benziodiazepinas nos transtornos de ansiedade. 2012. 
Disponível em: < http://www.psicologia.pt/artigos/textos/TL0352.pdf>. Acesso em: 10 mai. 2017.

GRASSI, L.T.V.; CASTRO, J.E.S. Estudo do Consumo de Medicamentos Psicotrópicos no Município de Alto Araguáia - MT. Artigo Científico. Cáceres: Faculdade do Pantanal, 2012.

GRUBER, J.; MAZON, L.M. A prevalência na utilização de medicamentos psicotrópicos no município de Mafra: um estudo retrospectivo. Saúde Meio Ambient., v. 3, n. 1, p. 44-50, 2014.

LOPES, L.M.B.; GRIGOLETO, A.R.L. Uso consciente de psicotrópicos: responsabilidade dos profissionais da saúde. Brazilian Journal of Health, v. 2, n. 1, 2013.

NOTO, A.R., et al. Análise da prescrição e dispensação de medicamentos psicotrópicos em dois municípios do Estado de São Paulo. Rev Bras Psiquiatr, v. 24, n. 2, p. 68-73, 2002.

NUNES, B.S.; BASTOS, F.M. Efeitos colaterais atribuídos ao uso indevido e prolongado de benzodiazepínicos. Saúde e ciência em ação, v.3, n.1, p.71-82, 2016.

ROCHA, B.S. da.; WERLANG, M.C. Psicofármacos na Estratégia Saúde da Família: perfil de utilização, acesso e estratégias para a promoção do uso racional. Ciência \& Saúde Coletiva, v.18, n.11, p.3291-3300, 2013.

TORRES, M.L.D. et al. Prescrição de psicotrópicos e especialidade médica: estudo em uma farmácia comercial no município do Maranhão. Revista Científica do ITPAC, v.7, n.4, Pub.4, 2014.

YONEYAMA, B.C.; MARUITI, A.M.P.; ESTEVES, R.Z. Um olhar sobre os usuários de medicamentos psicoativos acompanhados na atenção primária em saúde em Maringá Paraná. Espaço para a saúde, v.17, n.1, p. 114-120, 2016. 